\title{
Millimetre wavelength methanol masers survey towards massive star forming regions
}

\author{
T. Umemoto ${ }^{1}$, N. Mochizuki ${ }^{2}$, K. M. Shibata ${ }^{3}$, D.-G. Roh ${ }^{4}$, \\ and H.-S. Chung ${ }^{4}$ \\ ${ }^{1}$ Nobeyama Radio Observatory, Minamimaki, Minamisaku, Nagano 384-1305, Japan \\ email: umemoto@nro.nao.ac.jp \\ ${ }^{2}$ Institute of Space and Astronautical Science, Sagamihara, Kanagawa, 229-8510, Japan \\ ${ }^{3}$ National Astronomical Observatory, Mitaka, Tokyo 181-8588, Japan \\ ${ }^{4}$ Taeduk Radio Astronomy Observatory, Hwaam-Dong, Yusong-Gu, Daejon 305-348, Korea
}

\begin{abstract}
We present the results of a mm wavelength methanol maser survey towards massive star forming regions. We have carried out Class II methanol maser observations at $86.6 \mathrm{GHz}$, 86.9 GHz and 107.0 GHz, simultaneously, using the Nobeyama $45 \mathrm{~m}$ telescope. We selected 108 $6.7 \mathrm{GHz}$ methanol maser sources with declinations above -25 degrees and fluxes above $20 \mathrm{Jy}$. The detection limit of maser observations was $\sim 3 \mathrm{Jy}$. Of the 93 sources surveyed so far, we detected methanol emission in 25 sources $(27 \%)$ and "maser" emission in nine sources $(10 \%)$, of which thre "maser" sources are new detections. The detection rate for maser emission is about half that of a survey of the southern sky (Caswell et al. 2000). There is a correlation between the maser flux of $107 \mathrm{GHz}$ and $6.7 \mathrm{GHz} / 12 \mathrm{GHz}$ emission, but no correlation with the "thermal" (non maser) emission. From results of other molecular line observations, we found that the sources with methanol emission show higher gas temperatures and twice the detection rate of $\mathrm{SiO}$ emission. This may suggest that dust evaporation and destruction by shock are responsible for the high abundance of methanol molecules, one of the required physical conditions for maser emission.
\end{abstract}

Keywords. masers, surveys, ISM: molecules, radio lines: ISM, stars: formation

\section{Introduction}

It is known that class II methanol maser emission is a good tracer for the earliest stages of massive star formation. Extensive surveys of methanol maser emission at $6.7 \mathrm{GHz}$ have been carried out in the southern hemisphere (Val'tts et al. 1999; Caswell et al. 2000). Caswell et al. (2000) detected maser emission at $107 \mathrm{GHz}$ in 22 of 84 sources (26\%) and detected $157 \mathrm{GHz}$ emission from only four sources (5\%). However, no systematic surveys have been carried out in the northern hemisphere: Minier \& Booth (2002) have surveyed a limited sample of 23 maser sources in the northern hemisphere. They detected maser emission at $107 \mathrm{GHz}$ from six sources $(26 \%)$ and at $86.6 \mathrm{GHz}$ from two sources (9\%). In this paper, we present the results of a systematic millimetre methanol maser survey in the northern hemisphere.

\section{Observations}

Methanol emission of $7_{2}-6_{3} A^{-}$at $86.6 \mathrm{GHz}, 7_{2}-6_{3} A^{+}$at $86.9 \mathrm{GHz}$ and $3_{1}-4_{0} A^{+}$at 107.0 GHz were simultaneously observed using the $45 \mathrm{~m}$ telescope of the Nobeyama Radio Observatory. In addition, we have made observations of $\mathrm{SiO}(J=2-1, v=0)$ and $\mathrm{H}^{13} \mathrm{CO}^{+}$ $(J=1-0)$ lines simultaneously, to distinguish between maser and non-maser emssion by 
comparing the methanol data with thermal emission lines. We selected 108 sources of 6.7 $\mathrm{GHz}$ methanol maser sources with declinations $>-25$ degrees and fluxes $>20 \mathrm{Jy}$ from the source list of Szymczak et al. (2000), Menten (1991) and others. We used two $3 \mathrm{~mm}$ SIS receivers. The backend was a bank of eight acousto-optical spectrometers with $37 \mathrm{kHz}$ resolution $\left(0.10 \mathrm{~km} \mathrm{~s}^{-1}\right.$ at $\left.107 \mathrm{GHz}\right)$. The detection limit of our maser survey was $\sim 3 \mathrm{Jy}$, the same as for the southern survey by Caswell et al. (2000).

\section{Results and discussion}

So far we have surveyed 93 sources, with methanol emission detected in 25 sources $(27 \%)$ and "maser" emission in nine sources (10\%). Three "maser" sources, 18556+0136, $19110+1045$ and $19216+1429$, are new discoveries. Including previously known maser sources in the area we still need to survey, a total of 13 maser sources (14\%) have been detected. Our detection rate of maser emission is about half that of the southern sky survey (Caswell et al. 2000). However, $\mathrm{H}^{13} \mathrm{CO}^{+}$emission has been detected in only 48 sources $(52 \%)$ in our sample, because of uncertainties in the $6.7 \mathrm{GHz}$ maser positions and the smaller sizes of the emitting regions. If we consider only sources with detections of $\mathrm{H}^{13} \mathrm{CO}^{+}$emission, then the detection rate for maser emission is $25 \%$, the same as for the southern hemisphere sample.

We have compared the maser flux at $107 \mathrm{GHz}$ and at $6.7 \mathrm{GHz} / 12 \mathrm{GHz}$ including the southern survey data. The maser flux at $107 \mathrm{GHz}$ is about $(1-10) \%$ of the flux at 6.7 GHz, and there is a correlation between the maser flux at $107 \mathrm{GHz}$ and at $6.7 \mathrm{GHz}$ and also at $12 \mathrm{GHz}$. However there is no correlation with the "thermal" (non maser) emission. We found that sources with methanol emission show higher gas temperature (determined from our $\mathrm{NH}_{3}$ observations) and have twice the detection rate of $\mathrm{SiO}$ emission. In addition, sources with maser emission have wider thermal linewidths from $\mathrm{SiO}$ than from $\mathrm{H}^{13} \mathrm{CO}^{+}$. This may suggest that dust evaporation and destruction by shocks from outflows is responsible for the high abundance of methanol molecules, one of the required physical conditions of maser emission.

\section{References}

Caswell, J. L., Yi, J., Booth, R. S. \& Cragg, D. M. 2000, MNRAS, 313, 599

Menten, K. M. 1991, ApJ (Letters) 380, L75

Minier, V. \& Booth, R. S. 2002, A\&SA, 387,179

Szymczak, M., Hrynek, G. \& Kuss, A. J. 2000, A $\& A S, 143,269$

Val'tts, I. E., Ellingsen, S. P., Slysh, V. I., kalenskii, S. V., Otrupcek, R. \& Voronkov, M. A. 1999, MNRAS, 310, 1077 